\title{
MUTATIONS IN THE ALDH7A7 GENE CAUSE PYRIDOXINE-DEPENDENT SEIZURES
}

\section{TO THE EDITOR}

Recently, Lin and colleagues have reported a case of pyridoxine-dependent seizures in this journal'. The diagnosis was based upon the clinical criteria as formulated by Baxter ${ }^{2}$. In discussing the case the authors state that the underlying pathophysiology of the disorder is unknown and that no biochemical aids are available in establishing the diagnosis.

Indeed, for 50 years pyridoxine dependent epilepsy has been a clinical diagnosis based on the clinical effect of pyridoxine and confirmation of the diagnosis by a trial of pyridoxine withdrawal. However, in 2006 mutations in the ALDH7A1 gene have been found in patients with pyridoxine-dependent seizures ${ }^{3}$. Since then, mutations within this gene have been shown to be present in the majority of patients with a clinical diagnosis of pyridoxinedependent seizures ${ }^{3-6}$. These mutations cause a deficiency in $\alpha$-aminoadipic semialdehyde ( $\alpha$-AASA) dehydrogenase, indirectly leading to a secondary deficiency in pyridoxal-5-phosphate (P5P), which causes seizures. Administration of pyridoxine restores the P5P pool, thus controlling seizure activity ${ }^{3}$.

Another consequence of $\alpha$-AASA dehydrogenase deficiency is an accumulation of $\alpha$-AASA within the body, leading to increased plasma and cerebrospinal fluid levels $^{3} . \alpha$-AASA is excreted in the urine, thus leading to highly increased urinary levels in pyridoxine dependent patients with ALDH7A1 mutations. We and others have shown that urinary $\alpha$-AASA should be used as the biomarker for pyridoxine dependent seizures ${ }^{3-5,7}$.

In conclusion, in contrast to what Lin and co-authors have stated, the underlying pathophysiology and genetic mutations can be determined in the vast majority of patients with pyridoxine-dependent seizures. The diagnosis is no longer merely dependent on clinical criteria, but can be confirmed at the metabolite level by measuring $\alpha$ AASA in the urine of suspected patients. Ideally, urinary $\alpha$-AASA quantification and, if the biomarker is increased, subsequent DNA analysis should be performed in any child with suspected pyridoxine-dependent seizures. A trial of withdrawal in these patients should be omitted'.

\section{REFERENCES}

1. Lin J, Lin K, Masruha MR, Vilanova LC. Pyridoxine-dependent epilepsy initially responsive to phenobarbital. Arq Neuropsiquiatr 2007;65: 1026-1029.

2. Baxter P. Epidemiology of pyridoxine dependent and pyridoxine responsive seizures in the UK. Arch Dis Child 1999;81:431-433.
3. Mills PB, Struys E, Jakobs C, et al. Mutations in antiquitin in individuals with pyridoxine-dependent seizures. Nat Med 2006;12:307-309.

4. Plecko B, Paul K, Paschke E, et al. Biochemical and molecular characterization of 18 patients with pyridoxine-dependent epilepsy and mutations of the antiquitin (ALDH7A1) gene. Hum Mutat 2007;28:19-26.

5. Salomons GS, Bok LA, Struys EA, et al. An intriguing "silent" mutation and a founder effect in antiquitin (ALDH7A1). Ann Neurol 2007; 62:414-418.

6. Kanno J, Kure S, Narisawa A, et al. Allelic and non-allelic heterogeneities in pyridoxine dependent seizures revealed by ALDH7A1 mutational analysis. Mol Genet Metab 2007;91:384-389.

7. Bok LA, Struys E, Willemsen MA, Been JV, Jakobs C. Pyridoxine-dependent seizures in Dutch patients: diagnosis by elevated urinary alpha-aminoadipic semialdehyde levels. Arch Dis Child 2007;92:687-689.

Jasper V. Been, $M D^{\prime}$, Levinus A. Bok, $M D^{2}$, Michèl A.A.P. Willemsen, $M D P h D^{3}$, Eduard A. Struys, $P h D^{4}$, Cornelis Jakobs, $P h D^{4}$ 'Department of Paediatrics, Maastricht University Hospital, Maastricht, The Netherlands; ${ }^{2}$ Department of Paediatrics, Máxima Medical Center, Veldhoven, The Netherlands, ${ }^{3}$ Department of Paediatric Neurology, Radboud University Nijmegen Medical Center, Nijmegen, The Netherlands; ${ }^{4}$ Metabolic Unit, Department of Clinical Chemistry, VU University Medical Center, Amsterdam, The Netherlands Corresponding author: Levinus A. Bok, Máxima Medical Center, Department of Paediatrics, PO Box 7777, 5500 MB VeldhovenThe Netherlands (L.Bok@mmc.nl).

\section{THE AUTORS' REPLY}

We read with great interest and appreciate the comments made by Dr. Jasper V. Been et. al. about our article "pyridoxine-dependent epilepsy initially responsive to phenobarbital"1. In his letter, Dr. Jasper called our attention about the mutation in the ALDH7A1 gene as the cause of pyridoxine-dependent seizures.

In 2006 Mills et al. has conducted the initial studies concerning the mutations in ALDH7A1. In his work, the human gene ALDH7A1 was identified and mapped to locus $5 q 31$ (the locus of pyridoxine dependent epilepsy). This gene encodes antiquitin that has aldehyde dehydrogenase activity and when mutations occur, it abolishes the activity of antiquitin as a D1-piperideine-6-carboxylate (P6C) $-\alpha$-aminoadipic semialdehyde ( $\alpha$-AASA) dehydrogenase. The accumulating $\mathrm{P} 6 \mathrm{C}$ inactivates pyridoxal 5'-phosphate (PLP) a cofactor essential for normal metabolism of neurotransmitter ${ }^{2}$.

Sequencing of ALDH7A1 from individuals with pyridoxine dependent seizures (PDS) revealed homozygous and heterozygous mutations that were not detected in control subjects ${ }^{2}$. 
In the following years, the pathogenesis of PDS were further detailed, specially in 2007 when Plecko et al. reported the ALDH7A1 mutational analysis in 18 patients with PDS $^{3}$ and Kanno et al. revealed point mutation in regions of the ALDH7A1 gene in 4 out of 5 patients with PDS 4 .

Those articles also reported that $\alpha$-AASA dehydrogenase deficiency is characterized by increases in $\alpha$-AASA in urine, plasma and cerebrospinal fluid ${ }^{3,4}$. Further, Bok et al., measuring $\alpha$-AASA in urine and plasma by liquid chromatography-tandem mass spectroscopy in 12 Dutch clinically diagnosed patients with PDS revealed that $\alpha$-AASA was elevated in both urine and plasma in 10 patients ${ }^{5}$.

The information provided by those reports culminated in the statement by Dr. Jasper that quantification of the urinary Đ-AASA should be used as a biomarker of PDS, that, ideally, DNA analysis should be performed in any child with suspected PDS, and that a pyridoxine trial of withdrawal should be omitted.

The information brought by those articles concerning the pathogenesis of PDS is irrefutable. Unfortunately, the reports of Kanno et al. ${ }^{4}$; Bok et al. ${ }^{5}$ and the letter of Struys et al. ${ }^{6}$ were published, in their final form, few months after the submission of our article. Also unfortunately, the seminal articles of Mills et al. ${ }^{2}$ and Plecko et al. ${ }^{3}$ failed to be brought to our knowledge leading to inadvertent and unintentional un-accuracy in our article regarding the pathophysiology of PDS.

The statement that the pyridoxine-withdrawal test for making a definitive diagnosis of PDS should be replaced by DNA analysis and biochemical tests detecting urinary $\alpha$-AASA should, however, be considered with care. It must be taken into account that DNA analysis and diagnostic biochemical tests are not available or are not affordable in many countries. It also must be taken into account that PDS leading to neonatal status epilepticus is an emergency and when there is a clinical and electroencephalography suspicious of PDS, pyridoxine must be given. Therefore, in places where the ideal situation cannot be achieved, the final diagnosis may only be possible in the traditional and in the readily available way.
This point of view is shared by Kumar et al. ${ }^{7}$ that sustain that pyridoxine withdrawal test, wherein pyridoxine is withheld from a suspected PDS patient to observe recurrence of seizures that respond to pyridoxine is considered to be the gold standard for the diagnosis of PDS. He goes further when proposing that in families where the parents are not convinced about the need for life-long pyridoxine therapy, consideration should be given to undertaking an early "pyridoxine withdrawal test". This, according to his opinion, in addition to making a definitive diagnosis of PDS for the physician, also demonstrates the need for continuing pyridoxine therapy to the parents, thereby guarding against the possibility of loss to follow-up?.

\section{REFERENCES}

1. Lin J, Lin K, Masruha MR, Vilanova LCP. Pyridoxine-dependent epilepsy initially responsive to phenobarbital. Arq Neuropsiquiatr 2007;65:1026-1029.

2. Mills PB, Struys E, Jakobs C, et al. Mutations in antiquitin in individuals with pyridoxine-dependent seizures. Nature medicine 2006;12:307-309.

3. Plecko B, Paul K, Paschke E, et al. Bichemical and molecular characterization of 18 patients with pyridoxine-dependent epilepsy and mutations of the antiquitin (ALDH7A1) gene. Human Mutation 2007;28:19-26.

4. Kanno J, Kure S, Narisawa A, et al. Allelic and non-allelic heterogeneities in pyridoxine dependent seizures revealed by ALDH7A1 mutational analysis. Mol Genet Metabol 2007;91:384-389.

5. Bok L, Struys E, Willemsen MA, Been JV, Jakobs C. Pyridoxine-dependent seizures in Dutch patients: diagnosis by elevated urinary alphaaminoadipic semialdehyde levels. Arch Dis Child 2007;92:687-689.

6. Struys E, Jakobs C. Alpha-aminoadipic semialdehyde is the biomarker for pyridoxine-dependent epilepsy caused by alpha-aminoadipic semialdehyde dehydrogenase deficiency. Mol Genet Metabol 2007;91:405.

7. Kumar P, Gupta VK, Mishra D. Non-compliance in pyridoxine-dependent seizures and a way out. J Pediatr Child Health 2007;43:571-572.

\footnotetext{
Jaime Lin', Katia Lin ${ }^{2}$,Marcelo Masruha Rodrigues ${ }^{3}$, Luiz Celso Pereira Vilanova ${ }^{4}$

From the Departments of Neurology and Neurosurgery, Federal University of São Paulo, São Paulo, Brazil '. Postgraduate researcher, Division of Child Neurology'; Associate-Physician, Epilepsy Research and Treatment Unit ${ }^{3}$; Associate-Physician, Division of Child Neurology ; Professor and Chairman, Division of Child Neurology. Corresponding author: Dr. Jaime Lin, Division of Child Neurology Department of Neurology and Neurosurgery, Federal University of São Paulo, Rua Botucatu 720, 04023-900

São Paulo, Brasil (E-mail: linjaime@yahoo.com).
} 